\title{
Reported and Unreported Potentially Important Incidental Findings in Urgent Nonenhanced Abdominal CT for Renal Colic
}

\author{
Elena Bellonia $^{a}$ Stefania Tentoni $^{b}$ Ilaria Fiorina ${ }^{c}$ Chandra Bortolotto $^{d}$ \\ Olivia Bottinelli ${ }^{e}$ Michaela Cellina ${ }^{f}$ Daniele Gibelli ${ }^{9}$ Cristina Rosti ${ }^{a}$ \\ Lorenzo Predad, e Fabrizio Calliada ${ }^{\mathrm{f}, \mathrm{h}}$ Paola Scagnelli ${ }^{\mathrm{i}}$
}

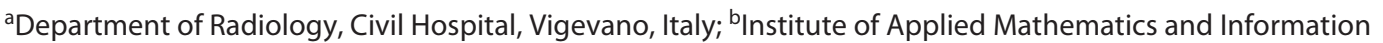
Technologies "Enrico Magenes", National Research Council, Pavia, Italy; 'Radiodiagnostic Department, Fondazione

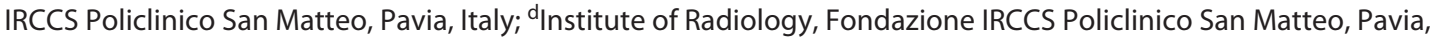
Italy; ' Department of Clinical-Surgical, Diagnostic and Pediatric Sciences, University of Pavia, Pavia, Italy; ${ }^{\mathrm{f}}$ Radiology Unit, Fatebenefratelli Hospital, ASST Fatebenefratelli Sacco, Milan, Italy; ${ }^{9}$ Department of Biomedical Sciences for Health, University of Milan, Milan, Italy; hDepartment of Radiology, IRCCS Fondazione Mondino, Pavia, Italy; 'Department of Radiology, Ospedale Maggiore, Lodi, Italy

\section{Highlights of the Study}

- Urgent nonenhanced abdominal CT is performed to rule out nephrolithiasis.

- Potentially important incidental findings are common in these abdominal CTs.

- "Satisfaction of search" may lead the radiologist to overlook important findings.

- Even in the urgent setting, the radiologist should report CT very carefully.

\section{Keywords}

Nephrolithiasis · Abdominal CT · Incidental finding ·

Reporting · Radiology

\begin{abstract}
Objective: The aim of the study was to retrospectively evaluate the prevalence of reported and unreported potentially important incidental findings (PIFs) in consecutive nonenhanced abdominal CTs performed specifically for renal colic in the urgent setting. Methods: One radiologist, blinded to the finalized report, retrospectively re-evaluated nonenhanced abdominal CTs performed from January to December 2017 on adult patients from the emergency department with the specific request of urgent evaluation for renal colic,
\end{abstract}

karger@karger.com www.karger.com/mpp

Karger $\stackrel{\text { ' }}{5}$
(C) 2021 The Author(s)

Published by S. Karger AG, Basel

This is an Open Access article licensed under the Creative Common Attribution-NonCommercial-4.0 International License (CC BY-NC) (http://www.karger.com/Services/OpenAccessLicense), applicable to the online version of the article only. Usage and distribution for commercial purposes requires written permission. searching for PIFs. Results: The CTs of 312 patients were included in the study. Thirty-eight findings were reported in 38 different CTs, whereas the re-evaluation added 47 unreported findings in 47 different CTs, adding to total of 85 findings $(27 \%)$. The difference in the proportion of reported and unreported PIFs between the original report and re-evaluation was significant $(p<0.001)$. No significant difference was found between the age of patients with and without reported findings. The proportion of potentially important findings did not vary significantly among the 3 shifts in the original report and in re-evaluation. The most frequent findings, both reported and unreported, were pleural effusion, lymphadenopathies, and liver nodules. Conclusions: Potentially important additional findings are frequently present in urgent nonenhanced abdominal CTs performed for renal colic, 
and many are not described in the finalized reports. Radiologists should take care not to underreport PIFs even in the urgent setting because of the possible consequences on the patient's health and in order to avoid legal issues, while at the same time satisfying the need for timely and efficient reporting.

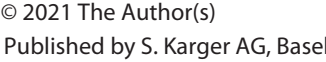

\section{Introduction}

A significant proportion (up to 15\%) of the general population is affected by nephrolithiasis, and therefore, acute renal colic is a frequent reason for accessing the emergency department. At present, nonenhanced abdominal CT is considered the reference standard imaging technique in stone detection [1]. Incidental findings can be defined as accidentally discovered abnormalities unrelated to the purpose of the performed examination. They should be distinguished into potentially important and potentially unimportant, in order to avoid unnecessary further diagnostic workup in the latter case, with consequent additional costs and patient anxiety [2]. As for potentially important incidental findings (PIFs), their recognition and reporting are fundamental in order to initiate prompt diagnosis and treatment, if necessary.

At our institution, a CT performed for renal colic covers all organs in the abdominal cavity and the pulmonary bases, with submillimeter spatial resolution; thus, incidental findings are a frequent issue in daily radiological practice. The aim of the study was to retrospectively evaluate the prevalence of reported and unreported PIFs in consecutive nonenhanced abdominal CTs performed specifically for renal colic in the urgent setting.

\section{Subjects and Methods}

\section{Study Population}

We retrieved nonenhanced abdominal CTs performed from January to December 2017 on adult patients in the emergency department with request for urgent evaluation for renal colic. One radiologist with 10 years of experience in CT re-evaluated the CTs, searching for PIFs. They were classified if they required further imaging and/or clinical workup, following the categorization proposed by Zalis et al. [3] for extracolonic findings in CT colonography (categories E3 and E4). The radiologist was blinded to the final report of the CT (for this purpose, the RIS-PACS administrator temporarily obscured the report) but had free access to the previous radiological examinations of the patient if present in the RISPACS. Also information was recorded on which shift the CT was performed and the report generated (i.e., morning shift 800 a.m.200 p.m.; afternoon shift $200-800$ p.m.; overnight shift 800 p.m.-
Table 1. General characteristics of the study patients $(n=312)$

\begin{tabular}{|c|c|}
\hline \multicolumn{2}{|l|}{ Variable } \\
\hline \multicolumn{2}{|l|}{ Sex } \\
\hline Male, $n(\%)$ & $202(64.7)$ \\
\hline Female, $n(\%)$ & $110(35.3)$ \\
\hline \multicolumn{2}{|c|}{$\begin{array}{l}\text { Age, years } \\
\text { mean } \pm \text { SD (range) }\end{array}$} \\
\hline All & $53.8 \pm 15.8(22-94)$ \\
\hline Males & $54.1 \pm 14.8(22-94)$ \\
\hline Females & $53.7 \pm 16.7(22-90)$ \\
\hline
\end{tabular}

800 a.m.). Interpretation of prospective images was performed by trained consultants in general radiology involved in emergency radiology shifts on rotation (generally once or twice a week); all reports were generated immediately after CT acquisition.

\section{Statistical Analysis}

The study variables were summarized using frequencies, range, and descriptive statistics (mean $\pm \mathrm{SD}$ ). The McNemar test was used to assess whether the proportion of patients, who either had reported or unreported findings, varied between the time of CT and the re-evaluation. The Wilcoxon rank-sum test was used to compare means of age between groups of patients with and without findings after testing for normality by using Shapiro-Wilk's test. Fisher's exact test was used to test whether the proportions of patients with PIFs varied among working shifts and age-groups. It was also used to test whether the proportion of unreported findings vis-à-vis the total of PIFs varied among shifts and age-groups.

All statistical tests were two-sided. $p$ values $<0.05$ were considered statistically significant. Data management and analyses were carried out using MATLAB R2017b Statistical Toolbox (The MathWorks, Inc., Natick, MA, USA).

\section{Results}

312 patients (202 males and 110 females), aged $53.8 \pm$ 15.8 years (range 22-94 years), were included in this study (Table 1). Obstructive nephrolithiasis was present in 284 (91\%) patients, of whom 186 were male. As shown in Table 2, 196 CTs were performed and reported in the morning shift, 98 in the afternoon shift, and 18 in the overnight shift. In the finalized reports, 38 reported PIFs in 38 different $\mathrm{CTs}(12 \%), 20$ in the morning, 16 in the afternoon, and 2 at night. Thirty-three findings (87\%) were in patients with nephrolithiasis. The most frequent findings were pleural effusion and/or parenchymal consolidation at the pulmonary bases (Fig. 1) and liver steatosis. The blinded retrospective evaluation confirmed all the 38 reported findings and added 47 unreported incidental findings in 47 different CTs ( 26 in the morning, 15 in the 
Table 2. CTs performed and potentially important findings proportions, at the time of examination and reevaluation, and by shift

\begin{tabular}{|c|c|c|c|c|}
\hline Variable & Time of examination & $p$ value & Re-evaluation & $p$ value \\
\hline \multicolumn{5}{|c|}{ All shifts, $n(\%)$} \\
\hline $\mathrm{CT}$ & $312(100)$ & \multirow{3}{*}{$<0.001^{\mathrm{a}}$} & $312(100)$ & \\
\hline PIF & $38(12.2)$ & & $85(27.2)$ & \\
\hline No PIF & $274(87.8)$ & & $227(72.8)$ & \\
\hline \multicolumn{5}{|c|}{ Morning shift, $n(\%)$} \\
\hline $\mathrm{CT}$ & $196(100)$ & \multirow{3}{*}{$0.331^{\mathrm{b}}$} & $196(100)$ & \multirow{3}{*}{$0.080^{\mathrm{b}}$} \\
\hline PIF & $20(10.2)$ & & $46(23.5)$ & \\
\hline No PIF & $176(89.8)$ & & $150(76.5)$ & \\
\hline \multicolumn{5}{|c|}{ Afternoon shift, $n(\%)$} \\
\hline CT & $98(100)$ & \multirow{3}{*}{$0.331^{\mathrm{b}}$} & $98(100)$ & \multirow{3}{*}{$0.080^{\mathrm{b}}$} \\
\hline PIF & $16(16.3)$ & & $31(31.6)$ & \\
\hline No PIF & $82(83.7)$ & & $67(68.4)$ & \\
\hline \multicolumn{5}{|c|}{ Overnight shift, $n(\%)$} \\
\hline $\mathrm{CT}$ & $18(100)$ & \multirow{3}{*}{$0.331^{\mathrm{b}}$} & $18(100)$ & \multirow{3}{*}{$0.080^{\mathrm{b}}$} \\
\hline PIF & $2(11.1)$ & & $8(44.4)$ & \\
\hline No PIF & $16(88.9)$ & & $10(55.6)$ & \\
\hline
\end{tabular}

PIF, potentially important incidental finding. ${ }^{a}$ McNemar test. ${ }^{b}$ Fisher's exact test.
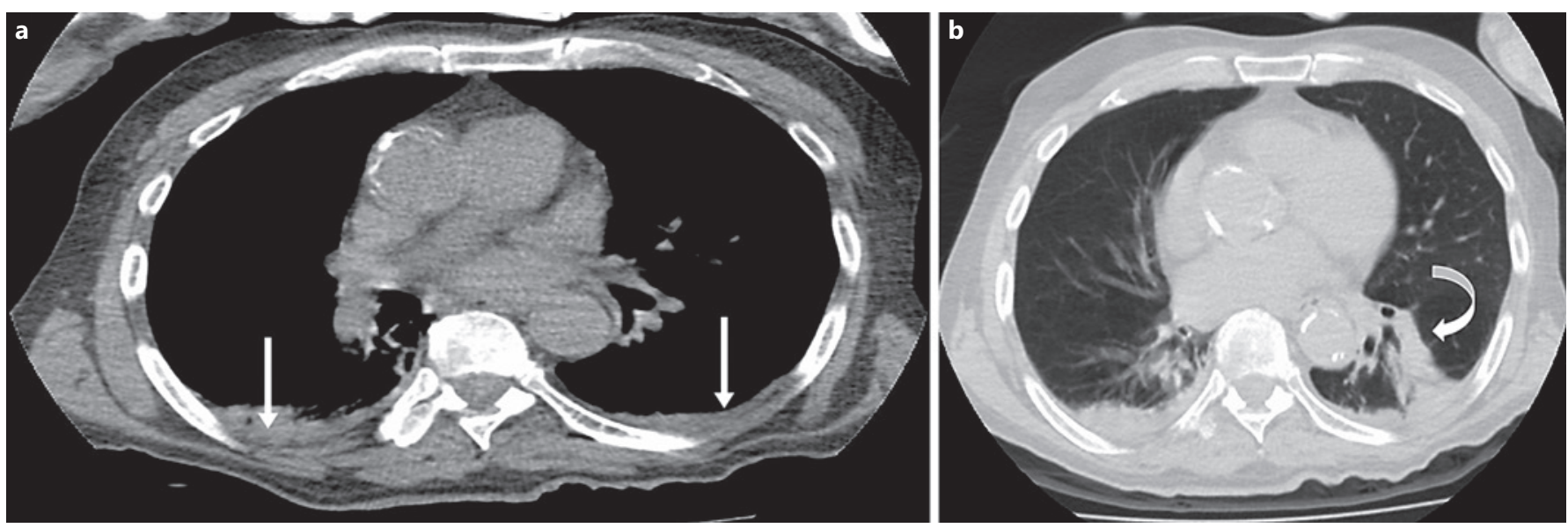

Fig. 1. Reported incidental findings in an 85-year-old male patient. Axial CT images with mediastinum (a) and lung (b) windowing showing bilateral pleural effusion (straight arrows) and parenchymal consolidation at the left pulmonary base (curved arrow).

afternoon, and 6 at night), adding to a total of 85 findings in 312 CTs, $27 \%$. Forty unreported findings (85\%) were in patients with nephrolithiasis. The most frequent unreported findings were focal hepatic alterations (Fig. 2) and lymph nodes larger than $1 \mathrm{~cm}$ in the short axis and/or round-shaped (lymphadenopaties). A particular finding was a lipoma of the right colon (Fig. 3). Table 3 summarizes reported and unreported findings.

The difference in the proportion of PIFs between the original report and re-evaluation was statistically significant $(p<0.001$, Table 2). No significant difference was found in the age of patients with and without findings, respectively ( $57 \pm 16$ and $53 \pm 16$ years for the 2 groups at time of examination, $p=0.195 ; 55 \pm 15$ and $53 \pm 16$ years after re-evaluation, $p=0.278$ ). The proportion of findings did not vary significantly among the 3 shifts in the original report and in the re-evaluation (Table 2). Table 4 shows incidence through age-groups, such as patients younger than 40 years, between 40 and 60 years, and those older than 60 years. No significant association was found between findings and age. 


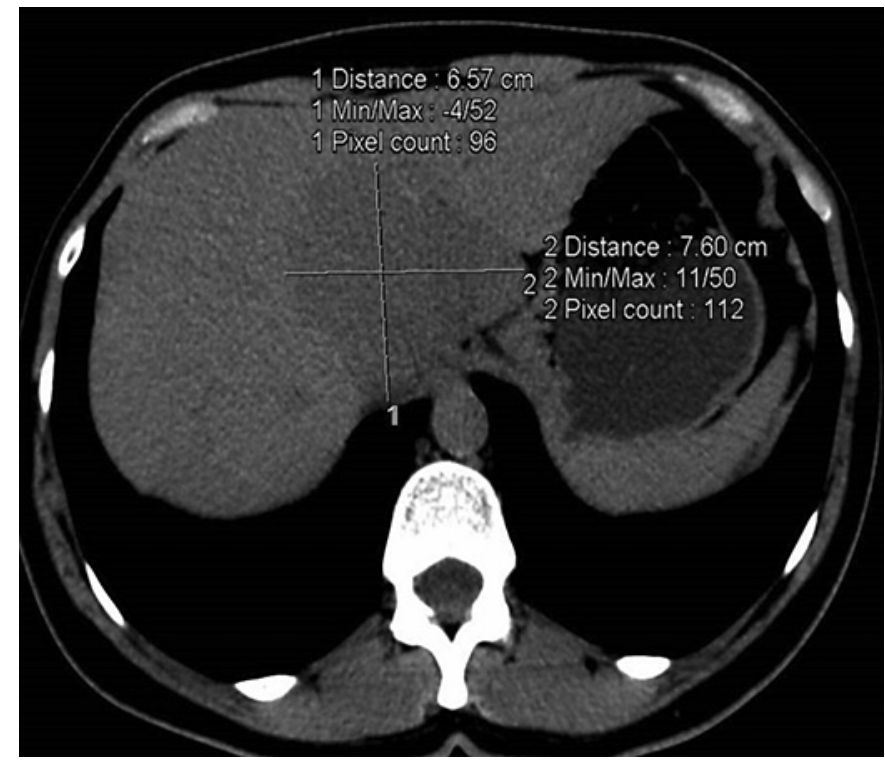

Fig. 2. Unreported incidental finding in a 43-year-old female patient. Axial CT image showing a hypodense $6.6 \times 7.6-\mathrm{cm}$ lesion at the hepatic dome.

\section{Discussion}

We observed a fair number of PIFs in nonenhanced abdominal CTs performed for renal colic in the urgent setting. In this situation, the report should be finalized as soon as possible at the end of the CT scan, as expected by physicians in the emergency department. As a result, the radiologist works under pressure and focuses on the specific query formulated for the examination, in our case to identify hyperdense obstructing renal stones causing the colic, possibly overlooking incidental findings. After nephrolithiasis has been either described or ruled out, the so-called phenomenon of "satisfaction of search" can occur, and other findings could be overlooked [4]. In our study population, the reported findings accounted for $12 \%$ of the total, whereas unreported ones were another $15 \%$. This implies that of the total incidental findings, $55 \%$ were not prospectively reported; at re-evaluation, incidental findings included all the 38 reported ones together with the 47 additional findings.

The issue of incidental findings in radiological examinations has been extensively studied, even if only partially in the urgent setting. The rates of PIFs vary greatly in various types of examinations, and this could be explained in part by the different characteristics of the populations studied. As for unreported findings, Quattrocchi et al. [5] found a significant number of unreported category E3 and E4 findings in the lumbar MRI (85\%), higher than ours; this is surprising because the examinations were not urgent. One possible explanation the authors gave was that musculoskeletal and neurological radiologists were focused on the spine only, and they concluded that a structured approach could improve the radiologists' detection rates. Though the setting of their study is quite different from ours, the use of a structured approach could be of benefit even in the emergency room [5]. Secchi et al. [6] evaluated incidental findings in cardiac MRI and concluded that only one-third of the important ones were included in the finalized reports; this proportion of unreported findings is in line with ours and raises the issue of the risks of hyperspecialization in radiology. Vertebral fractures are very frequently underreported by radiologists in nonurgent thoracic CTs, as stated by Williams et al. [7], especially in axial images; sagittal reformations are recommended to improve performance. Sverzellati et al. [8] studied urgent and nonurgent nonECG-gated thoracic CTs, searching specifically for significant cardiac findings, and found that two-thirds of them were not cited in the original reports. This conclusion is like ours, even if limited to cardiac findings and in a different population, and underscores that even radiologists trained in chest CT overlook significant cardiac findings [8].

Kuetting et al. [9] found a very high prevalence (80\%) and underreporting $(73 \%)$ of incidental cardiac findings in non-ECG-gated thoracic CT in intensive care unit patients. Their patients had similarities to ours, in that they needed a prompt CT report. They concluded that probably radiologists concentrated on the clinical question, overlooking incidental findings and experiencing the "satisfaction of search" phenomenon [9]. Contrary to our findings, Radhiana et al. [10] found that only $8 \%$ of significant findings were not mentioned in the report of urgent contrast-enhanced CT for abdominal blunt trauma. A possible explanation for this difference could be due to the use of a contrast medium.

Massersmith et al. [11] focused on urgent nonenhanced abdominal CT for renal colic but reported only on how often incidental findings were recorded and followed up by the emergency department. They found that approximately $25 \%$ of the reports had incidental findings of moderate or serious concern, but only a small proportion were highlighted in emergency department reports and followed up [11]. This conclusion is quite worrisome because it shows how often the importance of radiologists' reports is underestimated. A study on nonenhanced abdominal CTs performed in the urgent setting for suspected renal colic found that $12 \%$ of cases had additional 

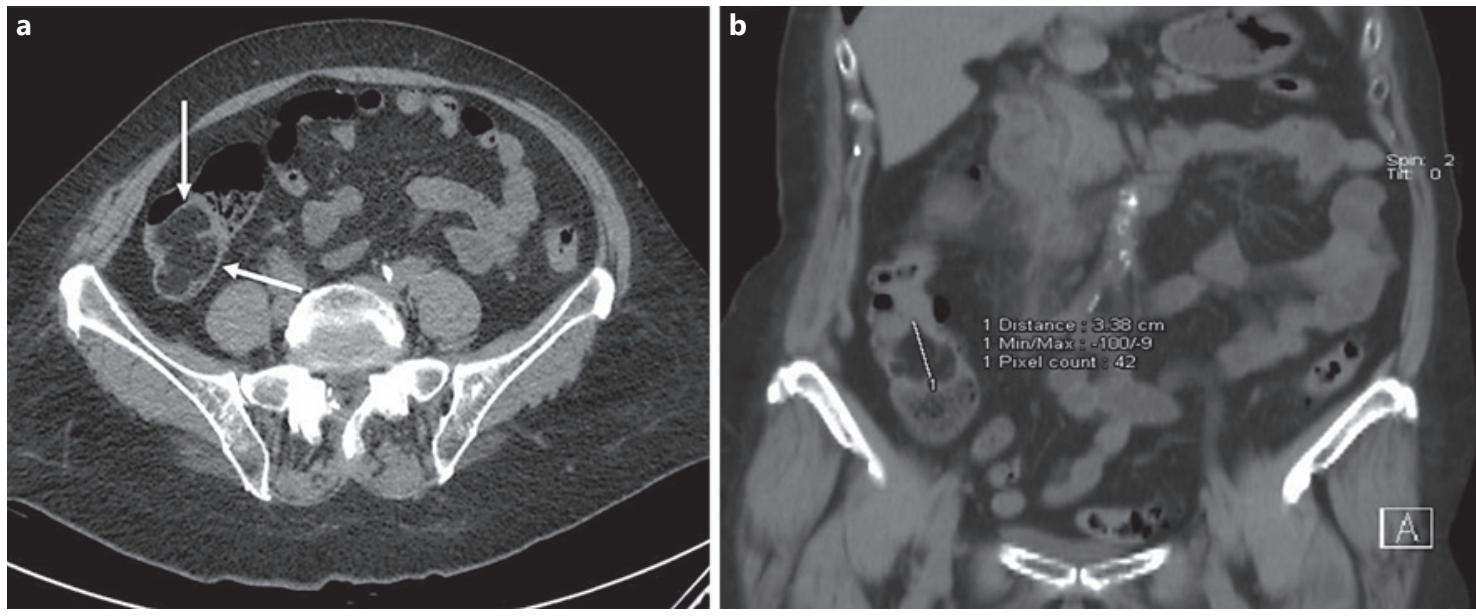

Fig. 3. Unreported incidental finding in a 50-year-old female patient. Axial (a) and coronal reformatted (b) CT images showing a markedly hypodense $3.4-\mathrm{cm}$ lesion in the lumen of the right colon (arrows), representing a lipoma.

Table 3. RPIF and UPIF

\begin{tabular}{lcc}
\hline & RPIF & UPIF \\
\hline Finding & 11 & \\
$\quad$ Pleural effusion & 9 & 8 \\
$\quad$ Liver steatosis & 7 & 6 \\
$\quad$ Pulmonary & 7 & 10 \\
Nodules $>1$ cm & 2 & 13 \\
$\quad$ Lymphadenopathies & 1 & 0 \\
Liver nodules & 1 & 0 \\
Mild pancreatitis & 0 & 4 \\
Mild cholecystitis & 0 & 2 \\
Adrenal nodules & 0 & 1 \\
Complex renal cysts & & \\
Colon lipoma & &
\end{tabular}

UPIF is the difference between the re-evaluation PIF and the RPIF. RPIF, reported potentially important findings; UPIF, unreported potentially important findings.

or alternative diagnoses. Again, only the radiologists' reports were evaluated, and possible unreported findings were not explored [12].

Another interesting finding of our study was that no significant difference was found between the age of patients with and without findings and in the proportion of findings through age-groups. Older patients are generally believed to have more incidental findings $[6,13]$; however, Williams et al. [7] highlighted a higher prevalence of vertebral fractures in younger patients.

Several limitations to our study should be mentioned. First, the number of CTs was quite low compared to oth-
Table 4. PIF occurrences through age-groups

\begin{tabular}{lrrrr}
\hline Variable & \multicolumn{2}{l}{ Age, years } & \multirow{2}{*}{$p$ value } \\
\cline { 2 - 4 } & 40 & $40-60$ & $60+$ & \\
\hline Original reports & & & & \\
$\quad$ PIF & 5 & 18 & 15 & $0.492^{\mathrm{a}}$ \\
$\quad$ No PIF & 52 & 139 & 83 & \\
Re-evaluation & & & & \\
$\quad$ PIF & 10 & 49 & 26 & $0.138^{\mathrm{a}}$ \\
$\quad$ No PIF & 47 & 108 & 72 & \\
\hline
\end{tabular}
test.

PIF, potentially important incidental finding. ${ }^{\text {a }}$ Fisher's exact

er reports in the literature. Second, the study was conducted in a single hospital, and this could have led to a bias in population selection. Also, we were not able to perform an analysis of the impact of the radiologists' experience on the rate of findings because of the small number of CTs reported by each staff radiologist. Moreover, because of the retrospective nature of the study, we were not able to perform a follow-up of the patients, and we missed information on prognostic significance of findings and outcome in those subjects (the majority) who did not undergo further evaluation in our hospital. Finally, the retrospective review was performed by a single radiologist who was unblinded to the purpose of the project; therefore, there could be unconscious bias toward reporting more findings. 


\section{Conclusion}

Potentially important additional findings are frequently present in urgent nonenhanced abdominal CTs performed for renal colic, and many of these are not described in the finalized reports. Radiologists should take care not to underreport PIFs even in the urgent setting because of the possible consequences on patients' health and in order to avoid legal issues, while satisfying the need for timely and efficient reporting.

\section{Statement of Ethics}

This retrospective observational study was approved by the San Matteo Ethics Committee (Study URO-TC, \#20180017958 approved on June 06,2018 ) and was carried out in accordance with the Declaration of Helsinki for experiments involving human subjects. The need for written informed consent was waived due to the retrospective nature of the study.

\section{Conflict of Interest Statement}

Chandra Bortolotto is a consultant for Bracco Imaging and Doc Congress. Fabrizio Calliada is a consultant for Bracco Imaging Italia, Hitachi Medical System Europe, Shenzhen Mindray Bio-Medical. The other authors declare that they have no conflict of interest.

\section{Funding Sources}

The authors did not receive any funding.

\section{References}

1 Miller NL, Lingeman JE. Management of kidney stones. BMJ. 2007;334(7591):468-72.

2 Berland LL, Silverman SG, Gore RM, MayoSmith WW, Megibow AJ, Yee J, et al. Managing incidental findings on abdominal CT: white paper of the ACR incidental findings committee. J Am Coll Radiol. 2010;7(10): $754-73$.

3 Zalis ME, Barish MA, Choi JR, Dachman AH, Fenlon HM, Ferrucci JT, et al. CT colonography reporting and data system: a consensus proposal. Radiology. 2005;236(1):3-9.

4 Berbaum KS, Franken EA, Dorfman DD, Rooholamini SA, Kathol MH, Barloon TJ, et al. Satisfaction of search in diagnostic radiology. Invest Radiol. 1990;25(2):133-40.

5 Quattrocchi CC, Giona A, Di Martino AC, Errante Y, Scarciolla L, Mallio CA, et al. Extra-spinal incidental findings at lumbar spine MRI in the general population: a large cohort study. Insights Imaging. 2013;4(3):301-8.
6 Secchi F, Lanza E, Cannaò PM, Petrini M, Sconfienza LM, Sardanelli F. Noncardiac findings in clinical cardiac magnetic resonance: prevalence in 300 examinations after blind reassessment. J Comput Assist Tomogr. 2013;37(3):382-6.

7 Williams AL, Al-Busaidi A, Sparrow PJ, Adams JE, Whitehouse RW. Under-reporting of osteoporotic vertebral fractures on computed tomography. Eur J Radiol. 2009;69(1):17983.

8 Sverzellati N, Arcadi T, Salvolini L, Dore R, Zompatori M, Mereu M, et al. Under-reporting of cardiovascular findings on chest CT. Radiol Med. 2016;121(3):190-9.

9 Kuetting D, Müller A, Feisst A, Luetkens J, Dabir D, Schild HH, et al. Incidental cardiac findings in non-electrocardiogram-gated thoracic computed tomography of intensive care unit patients: assessment of prevalence and underreporting. J Thorac Im. 2018;33(3): 168-75.
10 Radhiana H, Siti Kamariah CM, Mohd Nazli $\mathrm{K}$, Azian AA. Computed tomography (CT) of blunt abdominal trauma: the frequency of incidental findings, how it was documented in radiology report and the implication of these findings to acute trauma care. Med J Malaysia. 2014;69(1):46-8.

11 Massersmith WA, Brown DFM, Barry MJ. The prevalence and implications of incidental findings on ED abdominal CT scans. Am J Emerg Med. 2001;19:479-81.

12 Ahmad NA, Ather MH, Rees J. Incidental diagnosis of diseases on un-enhanced helical computed tomography performed for ureteric colic. BMC Urol. 2003;3:2.

13 Song JH, Beland MD, Mayo-Smith WW. Incidental clinically important extraurinary findings at MDCT urography for hematuria evaluation: prevalence in 1209 consecutive examinations. AJR Am J Roentgenol. 2012; 199(3):616-22. 